\title{
Emergence of Friedmann Equation of Cosmology of a Flat Universe from the Time-Energy Uncertainty Principle
}

\author{
Vernon Cooray $^{1 *}$, Gerald Cooray ${ }^{2}$, Farhad Rachidi ${ }^{3}$ \\ ${ }^{1}$ Department of Engineering Sciences, Uppsala University, Uppsala, Sweden \\ ${ }^{2}$ Karolinska Institute, Stockholm, Sweden \\ ${ }^{3}$ École polytechnique fédérale de Lausanne, Lausanne, Switzerland \\ Email: gerald.cooray@ki.se,farhad.rachidi@epfl.ch
}

How to cite this paper: Cooray, V., Cooray, G. and Rachidi, F. (2017) Emergence of Friedmann Equation of Cosmology of a Flat Universe from the Time-Energy Uncertainty Principle. Journal of Modern Physics, 8, 1979-1987.

https://doi.org/10.4236/jmp.2017.812119

Received: October 6, 2017

Accepted: November 24, 2017

Published: November 27, 2017

Copyright (c) 2017 by authors and Scientific Research Publishing Inc. This work is licensed under the Creative Commons Attribution International License (CC BY 4.0).

http://creativecommons.org/licenses/by/4.0/

\begin{abstract}
Friedmann equation of cosmology is based on the field equations of general relativity. Its derivation is straight-forward once the Einstein's field equations are given and the derivation is independent of quantum mechanics. In this paper, it is shown that the Friedmann equation pertinent to a homogeneous, isotropic and flat universe can also be obtained as a consequence of the energy balance in the expanding universe between the positive energy associated with vacuum and matter, and the negative gravitational energy. The results obtained here is a clear consequence of the fact that the surface area of the Hubble sphere is proportional to the total amount of information contained within it.
\end{abstract}

\section{Keywords}

Friedmann Equation, Expansion of the Universe, Vacuum Energy, Dark Energy, Time-Energy Uncertainty Principle, Holographic Principle, Hubble Sphere

\section{Introduction}

In an expanding universe, the speed at which objects recede from an observer increases as the distance to the object increases. There is a distance beyond which this speed becomes larger than the speed of light. This limiting distance is called the Hubble distance or Hubble radius and the Hubble constant is defined as the ratio of the speed of light to the Hubble radius, i.e. $H=c / R$ where $H$ is the Hubble constant (actually it varies with the age of the universe) and $R$ is the 
Hubble radius [1]. Therefore, there is a spherical region surrounding an observer beyond which the objects recede from the observer with a speed greater than the speed of light. The light emitted by objects located beyond the Hubble radius will never reach the observer. In other words, there is no causal connection between points that are located beyond the Hubble radius and the observer. It forms a boundary separating events visible at some time and the events that will never be visible [2] [3] [4].

In standard cosmology, the Hubble constant of an isotropic and homogeneous universe at any given cosmological time is given by [5] [6]

$$
H^{2}=\left[\frac{\dot{a}}{a}\right]^{2}=\frac{8 \pi G}{3}\left(\rho_{m}+\rho_{r}\right)+\frac{\Lambda c^{3}}{3}-\frac{k c^{2}}{a^{2}}
$$

In the above equation, $a$ is the scalar factor (which indeed is a function of cosmological time) of the universe, $\dot{a}$ is the derivative of $a$ with respect to time, $\Lambda$ is the cosmological constant, $\rho_{m}$ is the matter density (in $\mathrm{kg} / \mathrm{m}^{3}$ ), $\rho_{r}$ is the density of relativistic particles including radiation and neutrinos $\left(\mathrm{in} \mathrm{kg} / \mathrm{m}^{3}\right), k$ is a parameter that defines the curvature of the universe and $G$ is the gravitational constant. Defining $\rho_{k}=3 k c^{2} / 8 \pi G$ and $\rho_{0 \Lambda}=\Lambda c^{3} / 8 \pi G$, this equation can also be written as

$$
H^{2}=\left[\frac{\dot{a}}{a}\right]^{2}=\frac{8 \pi G}{3 c^{2}}\left[\frac{\rho_{0 m}}{a^{3}}+\frac{\rho_{0 r}}{a^{4}}+\rho_{0 \Lambda}-\frac{\rho_{k}}{a^{2}}\right]
$$

In the above equation, $\rho_{0 \Lambda}, \rho_{0 m}$ and $\rho_{0 r}$ are, respectively, the vacuum energy density (in $\mathrm{J} / \mathrm{m}^{3}$ ), matter (both normal and dark) energy density (in $\mathrm{J} / \mathrm{m}^{3}$ ) and the energy density of relativistic particles including radiation and neutrinos (in $\mathrm{J} / \mathrm{m}^{3}$ ) at the present time. Recent observations indicate that the expansion of the universe is accelerating and this accelerated expansion is attributed to the presence of vacuum energy [7] [8]. As the universe expands, the energy density associated with matter and radiation decreases while the vacuum energy density remains constant. In a vacuum energy dominated universe, the first two terms inside the bracket can be neglected in comparison to the vacuum energy term and the Friedmann equation reduces, in the case of a flat universe (i.e. $k=0$ ), to

$$
H_{0}^{2}=\left[\frac{\dot{a}}{a}\right]^{2}=\frac{8 \pi G \rho_{0 \Lambda}}{3 c^{2}}
$$

In the above equation, $H_{0}$ is the Hubble constant pertinent to a universe dominated by vacuum energy. Observe that in this case the Hubble constant becomes a true constant and the Hubble radius does not change with the cosmological time [5].

In this paper, we will demonstrate that Equation (2) (with $k=0$ ) and Equation (3) can be obtained as a consequence of the energy balance associated with the expanding universe. In a preliminary study conducted on this subject (uploaded as a pre-print; [9]) we have utilized a simple expression, based on the same concept to be exploited here, to estimate the magnitude of the vacuum energy density. In that preliminary study it was assumed that the derived expression is valid 
for all cosmological times. It indeed gives a reasonable estimate for the vacuum energy density if the currently available value of the Hubble radius is used in the expression. However, the results to be presented here show that the expression obtained in that preliminary study is correct only for cosmological times of a flat universe where the matter energy density is significantly smaller than the vacuum energy density.

\section{Main Hypothesis of the Paper}

In general relativity, the gravity is described as a curvature in space-time [10]. Matter or energy distorts or curves the space-time and the effect of the curved space-time is described as the gravity associated with the matter or energy. In a flat universe, the positive energy associated with matter and vacuum energy is balanced by the negative energy associated with their gravity [11] [12] [13] [14] [15]. In other words, the positive energy associated with matter and vacuum is balanced by the negative energy associated with the distortions it creates in the space-time. In this paper, it is assumed that the newly created space-time cannot be distorted or curved in a time less than about Planck time, and during this time, the positive energy associated with the vacuum and the matter located within this space-time do not experience any gravitational forces and as a consequence this positive energy cannot be balanced by the negative gravitational energy during this time. This also means that the newly created space-time cannot be curved or distorted instantaneously by the energy located in the new space but it takes a time in the order of Planck time for this to happen. As a consequence, the positive vacuum energy contained within the newly created space in an expanding universe is balanced by the negative gravitational energy only for time intervals larger than about $\gamma t_{p}$ where $t_{p}$ is the Planck time and $\gamma$ is a constant whose value is close to unity. For this reason, for times less than about $\gamma t_{p}$, the newly created space adds net positive energy to the universe and this net energy, as in the case of the creation of matter in quantum fluctuations, has to be extracted from empty space before it is being balanced by the negative gravitational energy. The amount of positive energy that can be extracted over a given time interval from empty space is dictated by the time-energy uncertainty principle [16].

\section{Friedmann Equation Pertinent to a Vacuum Energy Dominated Flat Universe Based on Energy Balance of the Expanding Universe}

Let us consider an observer located inside an expanding homogenous, isotropic universe with the following metric [5]:

$$
\mathrm{d} s^{2}=\mathrm{d} t^{2}-a^{2}\left[\mathrm{~d} x^{2}+\mathrm{d} y^{2}+\mathrm{d} z^{2}\right]
$$

The $x, y, z$ are orthonormal space coordinates, i.e. flat expanding space immersed in a flat Minkowski space. The proper distance between the observer and 
its Hubble horizon $R$ is given by

$$
R=a R_{0}
$$

In the above equation, $a$ is the scale factor and $R_{0}$ is the proper distance to some reference point at time $t_{0}$ [17]. In our analysis, we select $t_{0}$ to be a time when the universe is dominated by vacuum energy and $R_{0}$ is the proper distance to the Hubble horizon at that time. Let us consider a spherical volume of space with radius $R$ surrounding an observer located in an expanding universe. The net vacuum energy located inside this volume of space at any given time can be expressed as

$$
U=\frac{4}{3} \pi a^{3} R_{0}^{3} \rho_{0 \Lambda}
$$

The rate of change of the vacuum energy due to the creation of new space inside this volume is then given by

$$
\frac{\mathrm{d} U}{\mathrm{~d} t}=4 \pi a^{2} \dot{a} R_{0}^{3} \rho_{0 \Lambda}
$$

Now, at the Hubble horizon [2] [3] [4]

$$
R_{0} \dot{a}=c
$$

Using this relationship, Equation (7) can be written as

$$
\frac{\mathrm{d} U}{\mathrm{~d} t}=4 \pi c^{3}\left(\frac{a}{\dot{a}}\right)^{2} \rho_{0 \Lambda}
$$

The net positive energy, $\Delta U$, associated with the new space created within a time interval $\gamma t_{p}$ is then given by

$$
\Delta U=4 \pi c^{3}\left(\frac{a}{\dot{a}}\right)^{2} \rho_{0 \Lambda} \gamma t_{p}
$$

As mentioned in the previous section, this energy has to be borrowed within time $\gamma t_{p}$ from empty space and its magnitude is restricted by the time-energy uncertainty principle as follows:

$$
4 \pi c^{3}\left(\frac{a}{\dot{a}}\right)^{2} \rho_{0 \Lambda} \gamma t_{p} \approx h / 2 \pi \gamma t_{p}
$$

In the above equation, $h$ is the Planck constant. Observe that in writing down Equation (11), we are assuming that the newly created space located within the Hubble sphere acts, within a time interval in the order of Plank time, as a single quantum mechanical system. Now, the Planck time $t_{p}$ is given

$$
t_{p}=\sqrt{h G / 2 \pi c^{5}}
$$

Substituting this in Equation (11) we obtain

$$
H_{0}^{2}=\left(\frac{\dot{a}}{a}\right)^{2} \approx \frac{4 \pi G \gamma^{2} \rho_{0 \Lambda}}{c^{2}}
$$

This equation becomes identical to Equation (3) provided that $\gamma=\sqrt{2 / 3}$. Even if we have assumed that $\gamma=1$, Equation (11) still gives an order of magni- 
tude estimation of the Hubble constant for a given vacuum energy density. This also means that our assumption that the balance of positive vacuum energy by negative gravitational energy takes place at times on the order of Planck time is correct. It is Equation (11) that Cooray et al. [9] used to estimate the vacuum energy density using the current value of the Hubble constant as an input. For example the Hubble constant at the present time is about $22.4 \times 10^{-19}(\mathrm{~m} / \mathrm{s}) / \mathrm{m}$ (67.1 km/s/megaparsec) [18] [19]. If we substitute this number in Equation (11) we obtain $\rho_{0 \Lambda}=8 \times 10^{-10} \mathrm{~J} / \mathrm{m}^{3}$ which is close to the value of $6 \times 10^{-10} \mathrm{~J} / \mathrm{m}^{3}$ obtained from the measured rate of expansion of the universe [20]. As one can clearly see from the analysis presented here, this procedure is valid only in a universe which is dominated by vacuum energy. This assumption is approximately correct for our universe at the present time. Thus, the results presented here can also be considered as an alternative derivation of the vacuum energy density of free space. It is of interest, however, to investigate whether a similar analysis can be used to extract the Friedmann equation pertinent to a matter dominated flat universe. This is done in the next section.

\section{Friedmann Equation Pertinent to a Matter Dominated Universe Based on Energy Balance of the Expanding Universe}

As before, the new volume of space created in Planck time within the Hubble volume due to the expansion of the universe can be written as

$$
\Delta V=4 \pi c^{3}\left(\frac{a}{\dot{a}}\right)^{2} \gamma t_{p}
$$

Note that if the Hubble radius changes with time, the net volume of space inside the Hubble radius increases due to two reasons. First, it increases due to the creation of new space. Second, as the Hubble radius increases, it gains space that was located previously outside the Hubble sphere. In our analysis we are concerned only of the space that is being created fresh and that volume is still given by the above equation. The vacuum energy component associated with this newly created space is given by

$$
\Delta U_{\Lambda}=4 \pi c^{3}\left(\frac{a}{\dot{a}}\right)^{2} \gamma t_{p} \rho_{0 \Lambda}
$$

Now, let us consider the energy associated with the matter and radiation. The expansion of the universe does not change the net energy component associated with matter because the total amount of matter remains constant while the matter density decreases with the expansion. The energy associated with the photons decreases as the universe expands because of the stretching of the wavelength of the photons as the universe expands. As the universe expands, some of the matter ends up in the newly created space. According to our hypothesis the space-time cannot be curved or distorted in a time less than the Planck time and during this time the matter ended up in the newly created space will not expe- 
rience any gravitational force and hence the energy associated with it adds positive energy to the universe during this time. The hypothesis is depicted pictorially in Figure 1.

Consider a parcel of matter (represented by a dot in Figure 1) located inside the Hubble sphere. The energy associated with it is balanced by the negative gravitational energy (Stage I). At $t=0$, this parcel of matter ends up in newly created space-time which is not distorted and the universe gained an amount of energy equal to $m c^{2}$ where $m$ is the mass of the parcel of matter (Stage II). This new space-time becomes distorted in a time interval comparable to Planck time and after that time the net energy associated with the matter parcel goes to zero (Stage III). Thus the vacuum had to supply the energy necessary for the movement of the parcel of matter from Stage I to Stage II. Thus the net positive energy that is needed in the creation of new space, the volume of which is given by Equation (14) is,

$$
\Delta U_{M}=4 \pi c^{3}\left(\frac{a}{\dot{a}}\right)^{2} \gamma t_{p}\left\{\frac{\rho_{m}}{a^{3}}+\frac{\rho_{r}}{a^{4}}\right\}
$$

The quantity inside the curly bracket is the energy density of matter and the energy density of radiation at any given time. Thus the net energy that is needed in the creation of new space-time during Planck time is given by

$$
\Delta U=4 \pi c^{3}\left(\frac{a}{\dot{a}}\right)^{2} \gamma t_{p}\left\{\frac{\rho_{m}}{a^{3}}+\frac{\rho_{r}}{a^{4}}+\rho_{0 \Lambda}\right\}
$$

This energy is constrained by the time-energy uncertainty principle. Thus one can write
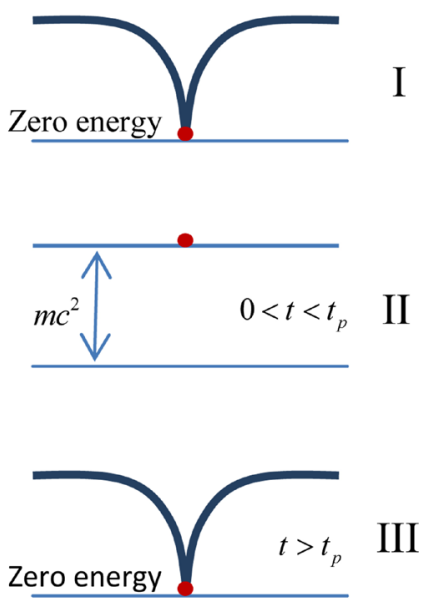

Figure 1. Stage I: A parcel of matter of mass $m$ (represented by the red dot) located inside the Hubble sphere and its energy is balanced by the negative gravitational energy; Stage II: This parcel of matter ends up inside newly created space-time which is not yet distorted. It requires a time interval on the order of Planck time for it to be distorted. The parcel of matter does not feel any gravitational forces during this time; Stage III: The newly created space-time gets distorted after a time interval comparable to the Planck time and the energy of the parcel of matter is balanced again by the negative gravitational energy. 


$$
4 \pi c^{3}\left(\frac{a}{\dot{a}}\right)^{2} \gamma t_{p}\left\{\frac{\rho_{m}}{a^{3}}+\frac{\rho_{r}}{a^{4}}+\rho_{0 \Lambda}\right\} \approx h / 2 \pi \gamma t_{p}
$$

As before, this equation can be written as

$$
H^{2}=\left(\frac{\dot{a}}{a}\right)^{2} \approx \frac{4 \pi G \gamma^{2}}{c^{2}}\left\{\frac{\rho_{m}}{a^{3}}+\frac{\rho_{r}}{a^{4}}+\rho_{0 \Lambda}\right\}
$$

With the value of $\gamma$ equal to the square root of $2 / 3$, this equation reduces to the Friedmann equation pertinent to a matter-dominated universe.

\section{Discussion}

The main assumption we have made in the derivation is that the total energy of a newly created volume of space remains positive for a time on the order of Planck time before the negative gravitational energy acts on the space volume and make the total energy zero. We have not shown that this is the case by appealing to fundamental physics. Observe also that in order to obtain Friedmann equation, it is necessary to consider the volume of space located inside the Hubble sphere. Considering any other arbitrary volume in the expanding universe would not allow us to obtain this equation. This indicates that the connection between the Friedmann equation, and hence the gravity in general, and the time-energy uncertainty principle, and hence quantum mechanics, is somewhat related to the Hubble radius or the cosmological horizon. The Equation (18), with $\gamma^{2}=2 / 3$, can be written as

$$
\left\{\frac{\rho_{m}}{a^{3}}+\frac{\rho_{r}}{a^{4}}+\rho_{0 \Lambda}\right\} \approx 3 h / 16 \pi^{2} c R^{2} t_{p}^{2}
$$

In the above equation, $R$ is the radius of the Hubble sphere. The left hand side of this equation gives the energy density of the universe. The net energy located inside the Hubble sphere can be obtained by multiplying the energy density as given by Equation (20) by the volume of the Hubble sphere. Thus the net energy located inside the Hubble sphere is given by

$$
U \approx h R / 4 \pi c t_{p}^{2}
$$

Now, consider a single photon of wavelength equal to the circumference of the Hubble sphere $2 \pi R$. The energy associated with such a photon is $h c / 2 \pi R$. The total number of such photons, $N$, associated with the energy located inside the Hubble sphere is given by

$$
N \approx R^{2} / 2 c^{2} t_{p}^{2}
$$

One can rewrite this equation as

$$
N \approx A / 8 \pi c^{2} t_{p}^{2}
$$

In the above Equation, $A$ is the area of the Hubble sphere. This equation can also be written as

$$
N \approx A / 4 l_{p}^{2}
$$


In the above equation $l_{p}$ is the Planck length as given by $l_{p}^{2}=h G / c^{3}$. This result shows that an energy equivalent to one photon of wavelength $2 \pi R$ is associated with an area of the Hubble sphere equal to $4 l_{p}^{2}$. Following the work of Bekenstein [21], the number $N$ can be interpreted as the number of bits of information located inside the Hubble sphere (see also [22]). Thus, each bit of information located inside the Hubble sphere is represented by an area equal to $4 l_{p}^{2}$ on the Hubble sphere. This result is identical to the one observed by Bekenstein [21] for black holes. Based on this information, Bekenstein estimated that the entropy of the black hole is proportional to the area of the black hole. The similarity of the results obtained here to the ones obtained by Bekenstein shows that the entropy of the Hubble volume is also proportional to its surface area. Indeed, Gibbons and Hawking [22] were the first to show that the surface area of the Hubble sphere has this remarkable property, i.e. Holographic principle [22]. That analysis shows that the principles of quantum mechanics when applied to the Hubble sphere can result in Friedmann equation. The results presented in this paper illustrate this connection for the first time using the time-energy uncertainty principle.

Observe that in deriving the Friedmann equations, in addition to the hypothesis described in Section 2, we have assumed that the universe is expanding. Equations of general relativity can indeed account for the expansion of the universe. Thus, we cannot claim that the derivation presented here is completely independent of the theory of gravity, because the above assumption of expanding universe is indeed based on the general theory of relativity.

\section{Conclusion}

It is shown that the Friedmann equation of cosmology pertinent to a flat, homogeneous and isotropic universe can be derived by assuming that the total energy of a newly created volume of space remains positive for a time on the order of Planck time before the negative gravitational energy acts on the space volume and make the total energy zero.

\section{References}

[1] Hubble, E.P. (1929) Proceedings of the National Academy of Sciences of the United States of America, 15, 168. https://doi.org/10.1073/pnas.15.3.168

[2] Dodelson, S. (2003) Modern Cosmology. Academic Press, London.

[3] Bergström, L. and Goobar, A. (1999) Cosmology and Particle Physics. Wiley International, New York.

[4] Melia, F. (2007) Monthly Notices of the Royal Astronomical Society, 382, 1917-1921. https://doi.org/10.1111/j.1365-2966.2007.12499.x

[5] Weinberg, S. (2008) Cosmology. Oxford University Press, Oxford.

[6] Friedmann, A. (1922) Zeitschrift für Physik, 10, 377-386. https://doi.org/10.1007/BF01332580

[7] Riess, A.G., et al. (1998) The Astronomical Journal, 116, 1009-1038. https://doi.org/10.1086/300499 
[8] Perlmutter, S., et al. (1999) The Astronomical Journal, 517, 565-586. https://doi.org/10.1086/307221

[9] Cooray, V., Cooray, G. and Rachidi, F. An Alternative Approach to Estimate the Vacuum Energy Density of Free Space. https://www.preprints.org/manuscript/201707.0048/v1

[10] Misner, C.W., Thorne, K.S. and Wheeler, J.A. (1973) Gravitation. W.H. Freeman and Company, New York, USA.

[11] Hawking, S. (2002) The Theory of Everything. New Millennium, London.

[12] Tryon, E.P. (1973) Nature, 246, 396-397. https://doi.org/10.1038/246396a0

[13] Pasachoff, J.M. and Filippenko, A. (2001) The Cosmos: Astronomy in the New Millennium. Harcourt College Publishers, Texas.

[14] Berman, M.S. (2009) International Journal of Theoretical Physics, 48, 3278-3286. https://doi.org/10.1007/s10773-009-0125-8

[15] Cooperstock, F.I. and Israelit, M. (1995) Foundations of Physics, 25, 631-635. https://doi.org/10.1007/BF02059009

[16] Wichmann, E.H. (1971) Quantum Physics, Berkeley Physics Course, Volume 4. McGraw-Hill, New York. https://en.wikipedia.org/wiki/Scale_factor_(cosmology)

[17] Riess, A., et al. (2005) The Astrophysical Journal, 627, 579. https://doi.org/10.1086/430497

[18] Sandage, A., et al. (2006) The Astrophysical Journal, 653, 843. https://doi.org/10.1086/508853

[19] Komatsu, E., et al. (2011) The Astrophysical Journal Supplement Series, 192, 18. https://doi.org/10.1088/0067-0049/192/2/18

[20] Bekenstein, J.D. (1974) Physical Review D, 9, 3292. https://doi.org/10.1103/PhysRevD.9.3292

[21] Susskind, L. (2008) The Black Hole War. Back Bay Books, Little, Brown and Company, New York.

[22] Gibbons, G.W. and Hawking, S. (1977) Physical Review D, 15, 2738-2751. https://doi.org/10.1103/PhysRevD.15.2738 